\title{
Del dicho al hecho: la narrativa de género del FMI y los derechos humanos de las mujeres
}

From words to deeds: the IMF gender narrative
and women's buman rights

\author{
Diane Elson ${ }^{1}$ \\ Universidad de Essex, Reino Unido \\ Corina Rodríguez Enríquez ${ }^{2}$ \\ UBA/CIEPP CONICET, Argentina
}

Revista Derechos en Acción ISSN 2525-1678/ e-ISSN 2525-1686

Año 6/NNo 18 Verano 2020-2021 (21 diciembre a 20 marzo), 275-310

DOl: https://doi.org/10.24215/25251678e483

Resumen: Este artículo analiza la narrativa de género del FMI y su coherencia con el tipo de políticas públicas y las acciones que el FMI recomienda a los países en sus acuerdos de préstamos. Analizando diversas dimensiones vinculadas con las brechas de desigualdad de género lla participación laboral, los derechos laborales, el trabajo doméstico y de cuidados no remunerados, la asignación de presupuesto a políticas que favorecen la igualdad, las metodologías para realizar análisis de impacto de género), se concluye que el FMI ha ido construyendo una narrativa de género que resulta estrecha e instrumental, y no contribuye en la práctica a promover los derechos humanos de las mujeres. Asimismo se advierte la contradicción persistente en las acciones que serían necesarias para

\footnotetext{
1 Profesora emérita de Sociología de la Universidad de Essex, Reino Unido, y expresidenta del Women's Budget Group, Reino Unido. La traducción de este artículo al español fue realizada por Victoria Sfriso y Natalia Barry.

2 Investigadora del Conicet en el Centro Interdisciplinario para el Estudio de Políticas Públicas (CIEPP). Miembro del Comité Ejecutivo de Mujeres por un Desarrollo Alternativo para una Nueva Era (DAWN).
} 
reducir la desigualdad y los estrechos márgenes de política pública que imponen los acuerdos con el FMI, así como las nocivas implicancias de las políticas que siguen exigiendo. El análisis se realiza con un foco específico en el caso de Argentina.

Abstract: This article analyzes the IMF's gender narrative and its consistency with the type of public policies and actions that the IMF recommends to countries in its lending agreements. By analyzing various dimensions linked to gender inequality gaps (labor participation, labor rights, unpaid domestic and care work, budget allocation to policies that favor equality, methodologies for gender impact analysis), we conclude that the IMF has been building a gender narrative that is narrow and instrumental, and does not contribute in practice to promoting women's human rights. We also highlight the persistent contradiction in the actions that would be necessary to reduce inequality and the narrow margins of public policy imposed by IMF's lending agreements, as well as the harmful implications of the policies that IMF continue to demand. The analysis is carried out with a specific focus on the case of Argentina.

\section{Introducción}

El Fondo Monetario Internacional (FMI) es miembro de las Naciones Unidas y se ha comprometido a apoyar los Objetivos de Desarrollo Sostenible (ODS), entre ellos, la lucha contra la desigualdad de género para alcanzar el ODS $5 .^{3}$ Así, el organismo ha comenzado a incursionar en la desigualdad de género y de ingresos como "cuestiones emergentes", y a desarrollar un asesoramiento explícito en políticas públicas de género. ${ }^{4}$

\footnotetext{
3 Ver, por ejemplo, FMI, Factsheet: The IMFand the Sustainable Development Goals, 2020. Disponible en inglés en https://www.imf.org/en/About/Factsheets/Sheets/2016/08/01/16/46/ Sustainable-Development-Goals

4 Emma Bürgisser y Sargon Nissan, "Positioning women's rights and gender equality in the macroeconomic policy environment", en Bretton Woods Project, The IMF and Gender Equality: A Compendium of Feminist Macroeconomic Critiques, (Bretton Woods Project, 2017).
} 
Sin embargo, el FMI se resiste a aceptar la idea de que tiene obligaciones en materia de derechos humanos, a pesar de que sus políticas tienen un impacto amplio y profundo en ellos. ${ }^{5}$ A pesar de ello, en 2017, en una carta dirigida al entonces Experto Independiente en derechos humanos de la ONU, Juan Pablo Bohoslavsky, el FMI afirmaba "promover indirectamente los derechos humanos" a través de su trabajo en pos de la igualdad de género. ${ }^{6}$ El presente artículo evalúa dicha afirmación con un foco especial en Argentina.

Para ello, en la sección 2 evaluamos la manera en que el FMI entiende a la igualdad de género. En la sección 3 revisamos cómo el FMI promueve la participación laboral de las mujeres pero sin considerar simultáneamente la dimensión de los derechos laborales. En la sección 4, revisamos la postura que el FMI asume respecto al trabajo doméstico y de cuidados no remunerado. En la sección 5, analizamos cómo operan las condicionalidades que el FMI impone a los países en relación con las brechas de desigualdad de género. En la sección 6 , repasamos la concepción del FMI respecto de los presupuestos con perspectiva de género. En la sección 7, analizamos la concepción de los modelos con los que el FMI evalúa el impacto de sus políticas. En la sección 8 realizamos una serie de sugerencias respecto de otras formas posibles de realizar estas evaluaciones. En la sección 9 analizamos para el caso específico de Argentina qué tipo de políticas debería promover el FMI para lograr efectivamente avances hacia la igualdad de género, y cómo el activismo feminista podría contribuir a este proceso. El artículo cierra con una sección de síntesis y conclusión.

5 Centro de Derechos Económicos y Sociales, Covid 19 and Recovering Rights. Briefing 12 Human Rights and the IMF's Covid Response, Diciembre 2020. Disponible en: www.cesr.org/ covid19

6 FMI, The IMF and Human Rights, submission letter to Juan Pablo Bohoslavsky, Independent Expert of the UN Human Rights Council, 27 de julio, 2017, disponible en inglés en: https:// www.ohchr.org/Documents/Issues/IEDebt/impactassessments/IMF.pdf 


\section{2. "La igualdad de género es una cuestión crítica desde el punto de vista macro"”: : cómo ve el FMI la igualdad de género en la actualidad}

Hasta hace poco, la igualdad de género era una cuestión invisible para el FMI. No la consideraba un objetivo que hiciera falta alcanzar, ni un factor que influenciara los resultados macroeconómicos, ni tampoco que estuviera influenciado por la política macroeconómica. La igualdad de género no estaba considerada como "macro-crítica", una categoría adoptada en 2012 por el FMI para evaluar qué cuestiones afectan a la economía a nivel macro y que, por tanto, son relevantes para el cumplimiento de su mandato. ${ }^{8}$ Sin embargo, en 2013, el organismo publicó una investigación demostrando lo "macro-crítico" que resultaba el incremento de la participación de las mujeres en la fuerza laboral, presentando evidencias de que esto podría aumentar el crecimiento económico y la productividad. ${ }^{\text {? }}$

Esta y otras investigaciones posteriores del FMI, así como trabajos operativos sobre la igualdad de género, allanaron el camino para la publicación, en junio de 2018, de una nota titulada "Cómo operacionalizar las cuestiones de género en el trabajo en los países" (Operationalising Gender Issues in Country Work). En ella, se afirma:

Promover el crecimiento económico y la estabilidad mundial requiere comprender los factores macro-críticos subyacentes, incluido el rol de la igualdad de género. La participación de las mujeres en el mercado laboral aumenta el tamaño y la reserva de talento de la fuerza

\footnotetext{
7 En lo que sigue, nombraremos como "macro-crítica" a esta consideración de las cuestiones de género como crítica desde un punto de vista macroeconómico.

8 Emma Bürgisser, The IMF and Gender Equality: Operationalising Change, (Brettons Woods Project, 2019).

9 Katrin Elborgh-Woytek, Monique Newiak, Kalpana Kochhar, Stefania Fabrizio, Kangni Kpodar, Philippe Wingender, Benedict Clements y Gerd Schwartz, Women Work and the Economy: Macroeconomic Gains from Gender Equity, Nota de discusión del equipo del FMI, SDN/13/10, 2013.
} 
laboral y contribuye a impulsar la productividad laboral y la producción. El empoderamiento económico de las mujeres - lo cual implica mayores niveles de escolarización, igualdad de derechos, mayor seguridad e inclusión financiera- también puede contribuir a mejorar la resiliencia económica y reducir la desigualdad de ingresos, apoyando así la estabilidad económica y el crecimiento sostenible. Por tanto, una mayor igualdad de género es fundamental para cumplir el mandato del FMI de promover la estabilidad económica. ${ }^{10}$

En la mencionada nota se constaba que se había producido un "valor añadido" a partir de 27 "programas pilotos de género" completados desde 2015 y que abarcaban países de las cinco regiones, los cuales incluían cuestiones de igualdad de género en las revisiones periódicas del Artículo IV (las consultas anuales entre el FMI y todos los gobiernos miembros), así como en los programas de préstamos. No obstante, el documento no recomendaba que las cuestiones de género se incorporaran siempre. En cambio, las recomendaciones eran que:

Los equipos deben cubrir las cuestiones de género de forma selectiva, ya sea cuando se consideren macro-críticas o cuando lo soliciten las autoridades. Si las cuestiones de género no son consideradas como macro-críticas, no se requiere su abordaje. Por lo tanto, no se espera una cobertura en profundidad de las cuestiones de género en los informes de todos los años, ni tampoco de todos los países. ${ }^{11}$

Esta nota no le proporciona al equipo del FMI una orientación clara sobre cómo determinar si las cuestiones de igualdad de género son macro-críticas y, en tal caso, cuáles lo son; por lo que depende en gran medida de las opiniones de los miembros

$10 \mathrm{FMI}$, How to Operationalize Gender Issues in Country Work, (FMI, 2018), 3 (traducción propia). Disponible en inglés en https://www.imf.org/en/Publications/Policy-Papers/ Issues/2018/06/13/pp060118howto-note-on-gender

11 FMI, op. cit. (nota 10), 4. 
del equipo del FMI y de las/os funcionarias/os y políticas/os con las/os que interactúan.

Aun cuando la igualdad de género es considerada "macrocrítica", el abordaje es profundamente acotado e instrumental, como un medio para lograr otros objetivos, no como un fin en sí mismo, tal como lo explicaremos en las próximas secciones. Hay cierta mención a la igualdad de derechos de las mujeres (como ocurre en la cita anterior) pero solo en referencia a los derechos comerciales, como el derecho a firmar contratos y a abrir una cuenta bancaria, que son importantes para la participación económica de las mujeres. Las leyes discriminatorias son consideradas un problema en la medida en que son obstáculos para que las mujeres contribuyan al crecimiento económico.

Lo que facilita esta visión instrumental es que el FMI no adopta un enfoque interseccional. La desigualdad de género se aborda por separado de la clase y la raza o etnicidad, por lo que el FMI no distingue distintos grupos de mujeres en relación con estas interseccionaldiades. Las mujeres son tratadas como un grupo homogéneo. Las directrices sobre los indicadores de género a incorporar en los informes sobre los países no sugieren el desglose según otras formas de desigualdad. ${ }^{12}$

En los países del Sur Global, donde la influencia del FMI cobra mayor importancia, la falta de un abordaje interseccional es especialmente relevante y grave. En el caso de Argentina, por ejemplo, la interrelación entre desigualdad de género y desigualdad socio-económica es evidente. Esto se puede mostrar con dos indicadores claves (incluso pensando solo en los términos estrechos en que lo hace el FMI, esto es, concentrándonos en la participación económica de las mujeres). La brecha de género en la participación laboral alcanza los 20 puntos porcentuales, siendo en promedio la tasa de actividad

12 FMI, op. cit. (nota 10). 
de los varones del 71,2 \% y la de las mujeres de 49,2 \%. ${ }^{13}$ Sin embargo, la tasa de actividad laboral de las mujeres que viven en hogares urbanos del quintil 5 (los de mayores ingresos) se eleva a $62 \%$, mientras la tasa de actividad laboral de las mujeres que viven en hogares urbanos del quintil 1 (los de menores ingresos) se reduce a $41,9 \%{ }^{14}$

Como contracara, la brecha en la dedicación de tiempo a actividades domésticas y de cuidado no remunerado es igualmente marcada entre las mujeres. Mientras las mujeres que viven en hogares del quintil 1 dedican en promedio 8,1 horas diarias al trabajo no remunerado, las mujeres que viven en hogares del quintil 5 dedican en promedio 4,5 horas diarias. ${ }^{15}$ La brecha en el peso de estas actividades entre mujeres resulta tan amplia como la brecha promedio entre mujeres y varones.

En Argentina, el abordaje interseccional también permite advertir la relevancia de mirar las diferencias de género en relación con la pertenencia o no a poblaciones indígenas, el lugar de residencia (especialmente relevante en un país de las dimensiones de Argentina y de enormes diferencias económicas territoriales), el origen migratorio, la identidad de género e incluso las diferencias generacionales. La ausencia de esta visión lleva a recomendaciones estándares que tienen luego severos problemas para servir a una población diversa y heterogénea.

\footnotetext{
13 Ministerio de Economía, Las brechas de género en Argentina. Estado de situación y desafíos, (Ministerio de Economía - Secretaría de Política Económica - Dirección Nacional de Economía, Igualdad y Género, 2020).

14 INDEC, Dossier estadístico por el 8M Día Internacional de la Mujer,(Instituto Nacional de Estadística y Censos, 2021).

15 Según información del Módulo de Trabajo no Remunerado y Trabajo Voluntario, de la Encuesta Permanente de Hogares del tercer trimestre de 2013. Esta es, hasta el momento, la única información de uso del tiempo de cobertura nacional disponible en Argentina.
} 


\section{EI FMI promueve la participación de las mujeres en la fuerza laboral, pero no sus derechos laborales}

El principal objetivo del FMI es aumentar la participación de las mujeres en la fuerza laboral. Se sugieren muchas políticas en esa dirección ${ }^{16}$, por ejemplo, cambios en los impuestos y en las prestaciones, un mejor acceso a los servicios de cuidado infantil, mejoras en las infraestructuras rurales y la eliminación de las restricciones legales que limitan la participación de las mujeres a sectores específicos de la economía y que restringen su acceso al crédito y a los derechos de propiedad. También se reclama un mejor acceso a la financiación y a la capacitación, y un mayor desarrollo de las redes de apoyo entre las empresarias.

Muchas de estas cuestiones fueron abordadas en los llamados "Informes país del Artículo IV". Por ejemplo, la consulta del Artículo IV de 2017 de la India ${ }^{17}$ condujo a la incorporación de políticas públicas recomendadas por el equipo del FMI en la estrategia del gobierno, en particular programas de capacitación específicamente segmentados por género y programas de alfabetización financiera. Estas recomendaciones se hicieron en base al análisis que concluyó que reducir la brecha de género en lo que refiere a la formación y al acceso a las finanzas formales aumenta la participación económica de las mujeres, lo cual podría impulsar el PBI de la India en cerca del $6 \%$. En este sentido, en India se ha creado un fondo nacional para otorgar microcréditos a las mujeres pobres a un costo asequible, y para ofrecer apoyo a actividades generadoras de ingresos.

Sin embargo, a medida que aumentan las tasas de participación femenina en la fuerza laboral, se sigue empujando a las trabajadoras de todo el mundo a puestos de trabajo feminizados, caracterizados por ingresos más bajos y condiciones de

\footnotetext{
16 Ver Elborgh-Woytek et al., op. cit. (nota 9).

17 FMI, How to Operationalize Gender Issues in Country Work, (FMI, 2018) Disponible en inglés en: http://www.imf.org/external/pp/ppindex.aspx
} 
trabajo más vulnerables. ${ }^{18}$ En los documentos del FMI se echa en falta cualquier mención a la mejora de los derechos de las mujeres en el empleo, y a su capacidad para formar grupos de apoyo colectivo y afiliarse a sindicatos. Tampoco se menciona el Programa de Trabajo Decente de la OIT. De hecho, el FMI lleva mucho tiempo impulsando políticas de flexibilización laboral, incluyendo políticas para mantener bajos los salarios, aumentar la flexibilidad en las prácticas de contratación y despido, y debilitar los convenios colectivos. Una revisión de los informes país del FMI, que cubre el período 2016-2020, muestra que 49 países de bajo ingreso y 40 de alto ingreso están promoviendo políticas de flexibilización laboral. ${ }^{19}$

Argentina constituye también un ejemplo de estas contradicciones. En el memorándum de entendimiento firmado entre el país y el FMI en junio de 2018 se incluyó el respectivo capítulo de género (el apartado C). En el mismo se afirma, en línea con lo que venimos señalando, que la visión de género del organismo es "[l]ograr que las mujeres argentinas puedan desarrollar todo su potencial no solo es una cuestión moral sino que también tiene sentido desde el punto de vista económico". ${ }^{20} \mathrm{Y}$ para lograr el desarrollo de este potencial se proponen, entre otras medidas, reducir los desincentivos tributarios a la participación laboral de las mujeres, promover acciones para reducir la brecha de género en los ingresos laborales, fortalecer los mecanismos de licencias vinculadas a responsabilidades de cuidado, ampliar la infraestructura para el cuidado de niños y niñas y la educación en la primera infancia, exigir que las empresas que cotizan en

18 Mary Borrowman y Stephan Klasen, "Drivers of Gendered Sectoral and Occupational Segregation in Developing Countries", 2 Feminist Economics, 2020, Vol. 26, 62-94.

19 Isabel Ortiz, Matthew Cummins, Jeronim Capaldo y Kalaivani Karunanethy, "The Decade of Adjustment: A Review of Austerity Trends 2010-2020 in 187 Countries", Documento de Trabajo Núm. 53, (The South Centre, IPD, y OIT 2015).

20 Ministerio de Economía y Banco Central, Memorandum de Políticas Económicas y Financieras, 2018, 10. Disponible en https://www.argentina.gob.ar/sites/default/files/ argentina_loi_-_mefm_-_tmou_-espanol3.pdf 
bolsa publiquen los datos de paridad en sus directorios, y avanzar medidas que enfrenten la violencia de género.

Sin embargo, el resto del memorándum compromete al gobierno argentino a las habituales reglas macrofiscales y las metas de equilibrio fiscal, que se riñen con la posibilidad de implementar políticas que avancen los compromisos sugeridos en el capítulo de género. Por ejemplo, el capítulo referido a la política fiscal establece compromisos que incluyen: racionalización del empleo público, reducir en un $15 \%$ el gasto en compras de bienes y servicios y posponer proyectos de obra pública no esenciales, todo lo cual resulta incompatible con la ampliación de políticas y servicios públicos que puedan contribuir a la participación económica de las mujeres. Esta contradicción se vincula asimismo con la posibilidad de reducir y redistribuir el trabajo doméstico y de cuidados no remunerado, tal como se señala en la próxima sección.

\section{El FMI reconoce el trabajo doméstico y de cuidados no remunerado pero solo como un obstáculo para el crecimiento económico}

En el documento del FMI del 2013 sobre mujeres, trabajo y economía, se indica que el trabajo no remunerado es una limitación a la capacidad de las mujeres de participar en el mercado laboral. El documento sugiere que un mejor acceso al cuidado infantil integral, asequible y de alta calidad, liberaría el tiempo de las mujeres para el mercado laboral. Recomienda proporcionarles subsidios a las madres trabajadoras para el cuidado de niños y niñas, "lo que impulsaría la participación femenina en la fuerza laboral sin distorsionar los incentivos del mercado laboral". ${ }^{21} \mathrm{De}$ este modo, se falla en reconocer que los servicios de cuidado infantil de alta calidad ofrecen beneficios significativos, independientemente de que las madres participen o no del mercado laboral, al mejorar tanto el desarrollo del niño o la niña como el

21 Elborgh-Woytek et al., op. cit. (nota 9). 
bienestar de la madre, ya que libera tiempo para actividades no relacionadas con el mercado, como la educación y la capacitación, y la participación en organizaciones comunitarias e instituciones políticas locales. Este documento tampoco aborda la manera en que se deben proveer los servicios de cuidado infantil.

Posteriormente, el FMI sí ha brindado consideraciones más detalladas sobre las políticas necesarias para reducir y redistribuir el trabajo doméstico y de cuidado no remunerado, al que describió como una "cuestión macro-crítica" y presentó una gran cantidad de datos sobre el alcance y la distribución por sexos de este trabajo. ${ }^{22}$ Sin embargo, en esta dimensión tampoco se tiene en cuenta la intersección entre género, clase y raza o etnia: por caso que los hogares acomodados del grupo racial o étnico dominante emplean a las mujeres pobres, especialmente a las provenientes de minorías raciales o étnicas, como trabajadoras remuneradas que luego en sus hogares realizan trabajos domésticos y de cuidados no remunerados. El FMI ha calculado que las ganancias económicas derivadas de las políticas de reducción y redistribución del trabajo no remunerado entre los géneros podrían sumar hasta el $4 \%$ del PBI. ${ }^{23}$ Según el organismo, el aumento del gasto público en servicios asistenciales tiene un rol que cumplir en esta cuestión.

Sin embargo, el gasto público puede adoptar diversas formas, que van desde la creación de servicios prestados por empleados/as bien capacitados/as del sector público con empleos decentes, hasta la subcontratación de empresas con ánimo de lucro que no optan por ofrecer empleos decentes, o de organizaciones comunitarias que no pueden permitirse ofrecer empleos decentes. El gobierno, por otro lado, puede no tener ningún rol y simplemente proporcionar transferencias monetarias $\mathrm{o}$ desgravaciones fiscales que ayuden a los hogares a contratar los

22 Cristian Alonso, Mariya Brussevich, Era Dabla-Norris, Yuko Kinoshita, y Kalpana Kochhar, Reducing and Redistributing Unpaid Work: Stronger Policies to Support Gender Equality, Documento de trabajo del FMI WP/19/225, 2019, 4.

23 Ibid., 2. 
servicios en el sector privado. La cantidad de trabajo doméstico y de cuidados no remunerado que hacen las mujeres puede disminuir, pero solo en tanto es reemplazado por empleos de mala calidad ocupados por otras mujeres, a menudo migrantes, que prestan servicios sustitutos. Además, que el empleo sea de mala calidad suele traducirse en una mala calidad del servicio, ya que el personal carece de formación y la rotación es alta.

En general, el FMI no apoya la expansión del empleo en el sector público y, en cambio, está muy a favor de las asociaciones público-privadas y de la subcontratación de servicios al sector privado en nombre de la eficiencia, por lo cual, se puede tener poca confianza en que la inversión gubernamental apoyada por el FMI en los servicios asistenciales vaya a producir servicios de alta calidad y empleos de alta calidad para los y las trabajadoras que los realizan.

Como se mencionó en la sección anterior, el memorándum de acuerdo entre Argentina y el FMI incluía una propuesta de ampliación de infraestructura para el cuidado de niños y niñas y de educación en la primera infancia. Esto resultaba compatible con el proyecto que el gobierno argentino de ese momento tenía previamente al acuerdo con el FMI, de construir 1000 jardines de infantes. Sin embargo, al término de la gestión de gobierno, se habían concluido solamente 107 de esas construcciones, lo que demuestra que, por un lado, el compromiso de ampliación de la infraestructura de cuidados era más declamativo que efectivo y, por el otro, que no se puede sostener un compromiso de inversión cuando simultáneamente se asumen compromisos de ajuste fiscal.

\section{Condicionalidad de género del FMI: invertir en el cuidado de niños y niñas, pero priorizar la consolidación fiscal}

Mientras que en el caso argentino el capítulo de género se reducía a un conjunto de recomendaciones sin ningún objetivo concreto, en otros casos el FMI ha empezado a incluir condiciones vinculadas a la igualdad de género en los acuerdos de 
préstamo. ${ }^{24}$ El programa de préstamo del FMI para Jordania de agosto de 2016 fue el primero en contener condicionalidades explícitas de género, al exigirle a Jordania que aumentara las tasas de participación femenina en la fuerza laboral mediante la mejora de la provisión de jardines de infantes. En el informe sobre Jordania del FMI, constaba:

Para fomentar el empleo femenino, las autoridades les exigen a las grandes empresas que ofrezcan jardines de infantes en sus instalaciones. Recientemente, se han presentado enmiendas a la ley laboral en el Parlamento para permitirles a las empresas compartir recursos y crear jardines de infantes conjuntos, a la vez que aumentan las sanciones a las empresas que no cumplan la ley; y han empezado a crear nuevos jardines de infantes en las zonas económicas especiales. El equipo sugirió que las enmiendas incluyan también la posibilidad de que las empresas subvencionen el cuidado de los niños y las niñas si hay impedimentos para crear jardines de infantes in situ o conjuntos. Esta política fomentaría el empleo en jardines de infantes privados y les proporcionaría a las familias y a las empresas una mayor flexibilidad. El equipo también recomendó la creación y promoción de jardines de infantes financiados con fondos públicos para trabajadoras y trabajadores de bajos ingresos ocupadas/os en PYMES. ${ }^{25}$

En respuesta, el gobierno declaró que:

Se requieren más modificaciones de la ley para permitir que las grandes empresas subvencionen el costo del cuidado de niños y niñas como alternativa al establecimiento de jardines de infantes. Hemos aprobado la creación de 6 jardines de infantes en zonas económicas especiales y seguiremos trabajando para aumentar la cantidad. Además, hemos permitido que algunas empresas situadas en zonas

24 Es interesante advertir que mientras las condicionales de política económica suelen tener implicancias concretas en caso de no cumplimiento (pudiendo llegar incluso a la finalización del acuerdo), las condicionales de género operan de manera no vinculante.

25 FMI, JORDAN. FMI Informe de país Núm. 17/231, 2017, 23. 
industriales $[\ldots]$ creen jardines de infantes conjuntos, y les hemos permitido a las grandes empresas que actualmente no tienen capacidad para ofrecer servicios de cuidado infantil en sus propias instalaciones crear jardines de infantes conjuntos en instalaciones seguras como medida temporal. En el caso de las empresas más pequeñas, se seguirá trabajando en la creación y promoción de jardines de infantes públicos y privados para trabajadoras/es de bajos ingresos ocupados/as en PYMES. Por último, trabajaremos en una campaña de concienciación pública y haremos un seguimiento de la directiva de 2014 del Primer Ministro sobre el establecimiento de jardines de infantes en todas las instituciones del sector público. ${ }^{26}$

Por lo tanto, aunque el aumento de la oferta de jardines de infantes reciba cierta atención, se trata de una oferta fragmentada en la que el sector privado desempeña un papel importante y no hay ninguna mención a la cantidad de dinero que el gobierno va a destinar a esto. Además, no hay nada que les proporcione servicios a las mujeres que trabajan en la economía informal. El foco en los jardines de infantes tiene por contexto continuar con la consolidación fiscal.

Luego, en enero de 2017, el FMI anunció que el programa de préstamos de 12000 millones de dólares destinado a Egipto contendría la condición de gastar 13 millones de dólares (una cantidad ínfima en el marco del programa global) a aumentar la disponibilidad de jardines de infantes, con el objetivo de incrementar la participación de las mujeres en la fuerza laboral, en un contexto general de consolidación fiscal. ${ }^{27}$ No se especificó cómo se debía gastar ese dinero.

Sin embargo, aunque el acceso a los servicios de guardería infantil es sin duda importante para promover una mayor

26 Ibid., 91.

27 FMI, Informe de país: República árabe de Egipto Núm. 17/17. 2017. 
participación femenina en el mercado laboral, no es suficiente. La tasa de desempleo femenino en Jordania es del 24,8 \%; lo que sugiere una escasez de puestos de trabajo que, por otra parte, la consolidación fiscal podría empeorar. Para apoyar la participación de las mujeres en la fuerza laboral, también se necesita protección social y otros servicios públicos, junto con más oportunidades de trabajo decente. En el marco de los requisitos del FMI para la reducción del gasto público global, las condicionalidades sobre aumentar la inversión en jardines de infantes pueden ir en detrimento de otros servicios públicos y de la protección social. ${ }^{28}$

Lo que adolece el enfoque de género del FMI es de una visión sistémica, tan esencial para comprender que una política aislada no puede por sí sola remover barreras estructurales. En América Latina, donde la agenda de género ha ido avanzando en varios países, y donde de hecho se han venido implementado - de manera tenue pero paulatina - diferentes políticas públicas tendientes a reducir las brechas económicas de género, la brecha de participación entre mujeres y varones se mantiene, según información de OIT, en más de 20 puntos porcentuales, la tasa de participación de las mujeres se encuentra estancada en el último quinquenio en torno al $50 \%$ y el desempleo ha crecido unos tres puntos porcentuales. ${ }^{29}$ Es decir, no es posible que una política aislada de fomento del empleo de las mujeres logre contrarrestar los efectos adversos de estrategias de desarrollo que, por estar basadas en la explotación intensiva de recursos naturales, generan poco empleo; o bien, contener las implicancias de contextos específicos de crisis económicas o más amplias (como la actual de la COVID-19).

\footnotetext{
28 Martha Alter Chen y Rachel Moussié, "Turning a blind eye to women in the informal economy", en Bretton Woods Project, The IMF and Gender Equality: A Compendium of Feminist Macroeconomic Critiques, (Bretton Woods Project, 2017).

29 OIT, Panorama Laboral de América Latina y el Caribe, (OIT, Oficina Regional para América Latina y el Caribe, 2020).
} 


\section{EI FMI promueve la presupuestación con perspectiva de género, pero lo hace para aumentar la eficiencia, no para garantizar los derechos económicos y sociales de las mujeres}

Desde una perspectiva feminista, la presupuestación con perspectiva de género conlleva el diseño y la implementación de los presupuestos gubernamentales de manera que apoyen, en lugar de socavar, la igualdad de género y los derechos de las mujeres, teniendo en cuenta los aspectos macroeconómicos generales del presupuesto, así como los programas e impuestos particulares. El enfoque feminista se basa en la crítica a la austeridad y a las reformas económicas neoliberales, y también al modo en que estas socavan los derechos de las mujeres. ${ }^{30}$ Desde mediados de los años noventa, los presupuestos sensibles al género han sido ampliamente respaldados en todo el mundo y promovidos por organizaciones internacionales y ONG. Los feminismos han contribuido a la elaboración de presupuestos con perspectiva de género con el desarrollo de herramientas de análisis, con asesoramiento y formación de funcionarios/as, representantes electos y ministros/as y en colaboraciones con las ONG que vigilan los presupuestos. Muchos gobiernos afirman haber incorporado la perspectiva de género a la hora de hacer sus presupuestos, aunque lo que entienden por ello varía enormemente. En muchos casos, lo que comenzó como una iniciativa feminista se ha visto diluido por parte de los gobiernos y las instituciones financieras internacionales, de modo que se ha perdido el potencial transformador. El modo en que el FMI ha promovido la presupuestación con perspectiva de género es un ejemplo de ello. ${ }^{31}$

Más tarde que temprano, el FMI descubrió la presupuestación con perspectiva de género y, en 2014, comenzó un

\footnotetext{
30 Diane Elson, Budgeting for Women's Rights; Monitoring Government Budgets for Compliance with CEDAW, (UNIFEM, 2006).

31 Diane Elson, "Gender budgeting", en Gunseli Berik y Ebru Kongar (ed.), Handbook of Feminist Economics, (Routledge, 2021).
} 
proyecto de investigación sobre este tema desde dentro del FMI, a cargo de Janet Stotsky, centrado en cómo una presupuestación sensible al género puede hacer que el gasto público sea más eficiente y más equitativo. Stotsky argumentó que el fundamento para presupuestar con perspectiva de género es que los presupuestos pueden considerar de manera inadecuada todos los beneficios económicos del desarrollo de las mujeres y la igualdad de género. ${ }^{32}$ Este enfoque fue bien acogido por el FMI y tiene respaldo como una herramienta para operacionalizar la cuestión de género en el ámbito del trabajo a nivel país: el FMI ha lanzado formaciones y capacitaciones en competencias específicas mediante talleres en los centros regionales donde brinda asistencia técnica, y también a través de asistencia técnica sobre la elaboración de presupuestos sensibles al género en Austria, Bahrein, Camboya y Ucrania, recientemente. ${ }^{33}$ El FMI resume las recomendaciones que se desprenden de un enfoque presupuestario con perspectiva de género de la siguiente manera: ${ }^{34}$

En las economías avanzadas, donde las diferencias entre varones y mujeres en materia de educación y sanidad han sido en gran medida eliminadas, un principal ámbito de desigualdad entre géneros es la participación en la fuerza laboral. De ahí que las recomendaciones incluyan a menudo la reducción de la carga fiscal para los y las trabajadoras secundarias (predominantemente femeninas), sustituyendo la tributación familiar por la individual, eliminando así los desincentivos relativos para la participación en el mercado laboral.

En los países de bajo ingreso y en vías de desarrollo, las recomendaciones suelen dirigirse a los vacíos tanto en las oportunidades como en los resultados. Esto incluye la equiparación de las tasas de escolarización de niños y

\footnotetext{
32 Janet Stotsky, Gender Budgeting: Fiscal Context and Current Outcomes, Documento de trabajo del FMI WP/16/149, (FMI, 2016).

33 FMI, op. cit. (nota 10), 7.

34 Idem
} 
niñas y el aumento de los niveles generales de educación. También se recomienda el aumento de la participación femenina en la fuerza laboral mediante inversiones en infraestructura y servicios de transporte para reducir los costos derivados del trabajo fuera del hogar [...] Asimismo, tener acceso a fuentes de electricidad y agua más cercanas al hogar puede liberar el tiempo de las mujeres para trabajar fuera de casa y permitirles integrarse en la economía formal. La provisión adecuada de servicios de salud ayudaría a reducir el tiempo dedicado a los cuidados informales. Hacer que las comunidades sean seguras para las mujeres aumenta su participación en el trabajo, la educación, la política y el ocio.

En los informes país del FMI consta cómo los gobiernos han adoptado la presupuestación con perspectiva de género, entre ellos Ruanda, Canadá, Islandia y Nigeria. Por ejemplo, el FMI considera que Ruanda ha tenido un éxito notable a la hora de abarcar las cuestiones de género en todos y cada uno de los niveles de operaciones gubernamentales, reduciendo la desigualdad de género al nivel más bajo del África subsahariana. No obstante, el análisis del equipo técnico del FMI se centró en los beneficios económicos de una mayor reducción de la desigualdad de género, que podría impulsar el crecimiento del PBI per cápita en medio punto porcentual. En concreto, un mayor acceso a servicios de salud y educativos de calidad y una mayor inclusión financiera podrían impulsar la participación laboral femenina en actividades económicas de mayor valor añadido, más allá de la agricultura. ${ }^{35}$

El foco está puesto en las medidas individuales de impuestos y gastos, no en el impacto en la brecha de género del presupuesto en su conjunto. Sin embargo, en una nueva partida, en la nota sobre "Cómo operacionalizar las cuestiones de género" se menciona la posibilidad de que los programas del FMI tengan un impacto negativo sobre la igualdad de género:

35 Ibid., 11. 


\begin{abstract}
Algunas políticas recomendadas por el equipo para apoyar el crecimiento y la estabilidad pueden tener un impacto diferencial de género que podría exacerbar la desigualdad entre géneros. Por ejemplo, los recortes presupuestarios en subsidios y programas sociales, los recortes en los salarios del sector público o el aumento de las tarifas de transporte podrían tener un mayor impacto en las mujeres. En estos casos, el equipo puede considerar una combinación alternativa de políticas para evitar esas externalidades negativas o - de no ser esto factible- sugerir algunas medidas de mitigación. ${ }^{36}$
\end{abstract}

Sin embargo, esto plantea la cuestión de qué métodos utilizará el FMI para determinar si las políticas recomendadas por el equipo podrían "exacerbar la desigualdad de género" y qué "combinación de políticas alternativas" considerará en tal caso. No hay pruebas de que el FMI haya analizado en la práctica las posibles repercusiones negativas, y el renovado interés por la consolidación fiscal como condición para los nuevos préstamos a los países en desarrollo en 2020 y 2021 sugiere que, en la práctica, las políticas alternativas no se tendrán demasiado en cuenta.

En el caso de Argentina, solo recientemente se ha comenzado a implementar una estrategia de presupuesto sensible al género a nivel del gobierno nacional, como una iniciativa autónoma y no por sugerencia o compromiso con el FMI. ${ }^{37}$ De hecho, el último presupuesto nacional es el primero en la historia en incluir explícitamente la perspectiva de género y diversidad. ${ }^{38} \mathrm{Si}$ bien esta experiencia resulta un avance innegable, lo cierto es que por ahora la perspectiva se reduce a identificar partidas que tienen potencialidad para reducir las brechas de desigualdad,

\footnotetext{
37 Vale mencionar que el presupuesto 2019, elaborado bajo la vigencia del acuerdo Stand by con el FMI, no incluyó ninguna referencia a la necesidad de incorporar la perspectiva de género en el presupuesto.
}

38 Sobre esta experiencia puede verse Ministerio de Economía, Presupuesto 2021: el primer presupuesto con perspectiva de género y diversidad. Una herramienta de transformación para cerrar brechas de desigualdad, (Ministerio de Economía - Secretaría de Política Económica Dirección Nacional de Economía, Igualdad y Género, 2020). 
con criterios teóricos (y por cierto bastante amplios), y sin que medien evaluaciones concretas de estos impactos. Si bien el etiquetado en el último presupuesto puede ser una buena plataforma, su potencial transformador dependerá de que se sofistique el mecanismo de manera de sustentarse en evaluaciones efectivas del impacto de las políticas, se institucionalice y se apoye en una formación sólida de quienes lo construyen en las diferentes dependencias del Estado. Caso contrario, corre el riesgo de diluirse como las iniciativas promovidas por el FMI. ${ }^{39}$

\section{Cómo evalúa el FMI el impacto de género de las políticas: los peligros de los modelos económicos convencionales}

El FMI ha desarrollado modelos econométricos que evalúan el impacto distributivo y macroeconómico de sus prescripciones de políticas. Gradualmente, va extendiendo estos modelos para estudiar los impactos desde una perspectiva de género $\mathrm{y}$, en 2017, los aplicó en Argentina e Irán para analizar impactos de posibles reformas impositivas y medidas destinadas a reducir la brecha entre géneros y la discriminación. ${ }^{40}$ El modelo, que se recomienda en la nota "Cómo operacionalizar las cuestiones de género en el ámbito del trabajo en los países", utiliza un modelo de equilibrio general dinámico y estocástico por el cual todos los mercados son transparentes (léase, la oferta y la demanda se equilibran en todos ellos y cualquier tipo de desempleo es temporario y de subsana por sí mismo); maridos y esposas en los hogares toman todas las decisiones relevantes de manera conjunta y armoniosa (es decir que, en los hogares, no hay más que cooperación siempre, jamás hay conflicto ni desigualdad); el trabajo doméstico y de cuidado no remunerado no

39 Un ejemplo de la necesidad de mejorar y fortalecer la herramienta de presupuesto sensible al género en Argentina, es como su aplicación al presupuesto 2021 no fue capaz de detectar el impacto negativo que tendría sobre las mujeres la eliminación de asignaciones presupuestarias para mantener el Ingreso Familiar de Emergencia (IFE).

40 FMI, Pursuing Women's Economic Empowerment, Background Note for the G7, 2018, 23. 
está explícitamente incluido en el modelo, pero si las mujeres trabajan en el mercado laboral, se asume que hay un "costo de utilidad", que "se relaciona con la dificultad de coordinar las múltiples tareas domésticas, como el mantenimiento del hogar y la crianza de niños y niñas"; no hay servicios del sector público, "el gobierno recauda impuestos a los ingresos personales, contribuciones sociales, impuestos al consumo e impuestos a las sociedades; y realiza transferencias de efectivo destinadas a hogares, pensiones y jubilaciones y bienes producidos por el sector formal"; no hay déficit presupuestario, pues la recaudación fiscal es igual al gasto público; y la economía se mueve ágilmente de un punto de equilibrio al otro, con demanda agregada y con una oferta equivalente a la demanda. ${ }^{41}$ Es evidente que este modelo deja de lado todos los temas que han preocupado a la crítica feminista de los análisis y prescripciones de políticas del FMI, como el modo en que la consolidación fiscal tiende a aumentar el trabajo no remunerado que deben hacer las mujeres.

En Argentina, el modelo se usó para evaluar el impacto desde una perspectiva de género en caso de reducir las contribuciones a la seguridad social de empleadores/as y empleados/as en $10 \%$ del salario bruto (sin incluir las contribuciones destinadas a la salud, a las llamadas "obras sociales") mientras que, al mismo tiempo, se aumenta el impuesto a los ingresos personales de personas físicas mediante el recorte de las llamadas "deducciones especiales" en $40 \%$. El costo directo por el recorte de las contribuciones a la seguridad social se estimó en una pérdida de recaudación de 1,8\% del PBI, mientras que se estimó que el recorte a las deducciones especiales aumentaría la recaudación adicional en 1,5\% del PBI. ${ }^{42}$ Estas propuestas implican que "se necesitará un gasto público menor para cubrir

41 Lisa Kolovich, Vivian Malta, Marina Mendes Tavares, "Addressing Gender Issues in Argentina's Labour Market, Appendix 1". En FMI, Argentina: Selected Issues. IMF Country Report Núm. 17/410, 2017.

42 Paolo Dudine, Ricardo Fenochietto y Vivian Malta, "How To Reduce Argentina's Tax Wedge". En FMI, Argentina: Selected Issues. IMF Country Report No. 17/410, 2017, 11. 
el costo de la reforma tributaria si se quiere, al mismo tiempo, seguir reduciendo el déficit". ${ }^{43}$

Se esperaba que las reformas del FMI beneficiaran a las mujeres por la provisión de inventivos atractivos que las motivaran a ingresar al mercado laboral formal gracias a las menores contribuciones sociales que se extraerían de su salario. "Después de la reforma, las mujeres trabajarían más horas en el sector formal (un $12 \%$ más)" y "el salario real promedio aumentaría en $3 \%$ para las mujeres y $2,7 \%$ para los varones, lo cual reduciría la brecha promedio del salario entre géneros"; y además, en el largo plazo "el PBI aumentaría en un 1,2\%" ${ }^{44}$ Estas conclusiones dependen de la ausencia de otras restricciones, posiblemente más vinculantes, a la participación de las mujeres en la fuerza laboral, como la falta de servicios adecuados de transporte y de cuidados, o la de puestos laborales en el sector formal, especialmente aquellos que posibilitan a trabajadores y trabajadoras equilibrar su trabajo remunerado con las responsabilidades de cuidado a su familia.

Para ilustrar algunas de estas restricciones estructurales al empleo de las mujeres en Argentina, se puede señalar que el $16,5 \%$ del total de las mujeres ocupadas y el $21,5 \%$ del total de las asalariadas se ocupan en el servicio doméstico, un sector de actividad donde el nivel de informalidad aún supera el $65 \%$ y donde los salarios se ubican entre los más bajos del mercado, con lo cual ni una rebaja en las contribuciones a la seguridad social, y mucho menos en las deducciones en el impuesto a los ingresos personales es probable que tengan impacto. Asimismo, como ya se comentó anteriormente, el peso del trabajo doméstico y de cuidados no remunerados sigue siendo una barrera persistente a la participación económica de las mujeres que no se ve afectada por este tipo de incentivos monetarios. De hecho, parte de la intensidad del uso del tiempo en este tipo de actividades a

\footnotetext{
43 FMI, Informe de país de Argentina Núm. 17/409, 2017, 32.

44 Lisa Kolovich, Vivian Malta y Marina Mendes Tavares, "Addressing Gender Issues in Argentina's Labour Market, Appendix 1", en FMI, Argentina: Selected Issues. IMF Country Report Núm. 17/410, 2017, 81.
} 
cargo de las mujeres se vincula con la dificultad para acceder a servicios de cuidado. Hacia el año 2016, la tasa de asistencia a establecimientos de educación inicial alcanzaba para el total del país a 40,6 \% en el caso de niños y niñas de 3 años; 9,8 \% para los de 2 años y apenas $2 \%$ para los de menos de 1 año. ${ }^{45}$

En definitiva, no hay certeza de que tendrá lugar la respuesta de oferta prevista por el modelo. Lo que sí se prevé es la pérdida de recaudación impositiva, lo cual puede poner en peligro el financiamiento disponible para las transferencias de efectivo y los servicios públicos. ${ }^{46}$

El análisis de impacto de género del FMI también estudiaba la provisión de subsidios para el cuidado infantil para las mujeres de ingresos bajos y medios que trabajan en el sector formal, y encontró que esto incrementaría la participación femenina en el sector de empleo formal, reduciría la brecha del salario entre géneros y generaría mayor crecimiento económico. Se encontró que estas políticas serían más efectivas que las reformas impositivas en cuanto al aumento de la cantidad de horas de trabajo por parte de mujeres en el sector formal y en cuanto al incremento del crecimiento económico. Se enfatiza así que, en el largo plazo, un mayor impuesto al ingreso por parte de las mujeres empleadas en el sector formal podría compensar ampliamente al gobierno por el costo del subsidio para el cuidado infantil (si se asume que habrá más puestos de trabajo disponibles en el sector formal). Sin embargo, estas políticas ignoran las diferencias de clase entre las mujeres: si solo se destina a las mujeres del sector formal, deja de lado a millones de mujeres de bajos ingresos que trabajan en el sector informal y que también necesitan disponer de cuidado infantil. Es más, no existe un análisis en cuanto a cómo funcionarían estos subsidios destinados al cuidado infantil: el modelo implica que habría más transferencias de efectivo que se destinarían a ese

\footnotetext{
45 Unicef - Cippec, Mapa de la Educación Inicial en Argentina, (UNICEF - CIPPEC, 2018).

46 Bürgisser, op. cit. (nota 8)
} 
fin, pero no detalla quiénes proveerían el servicio, si empresas de cuidado infantil pertenecientes al sector formal, o personal doméstico del sector informal. Tampoco se presta atención a la necesidad de personal bien capacitado y bien remunerado que provea este trabajo de cuidados remunerado. Esta política puede profundizar la desigualdad entre mujeres que pagan por el cuidado de sus niños y quienes son remuneradas por proveer ese servicio.

Irónicamente, el análisis de impacto verificó que si la brecha del salario entre géneros se redujera en 6 puntos porcentuales, esto tendría un impacto mayor sobre el crecimiento económico que las reformas impositivas o el subsidio para el cuidado infantil. Quienes realizaron el análisis esperaban que la reducción de las brechas de género en los ingresos laborales se lograra mediante la reducción de las barreras legales al acceso al empleo por parte de mujeres y mediante el lanzamiento de campañas de concienciación sobre la desigualdad de género. Pero, al mismo tiempo, el FMI recomendó que las llamadas "distorsiones" del mercado laboral se redujeran a través de:

(i) dinamizar los procedimientos de despido...; (ii) reducir el nivel requerido de indemnizaciones por despido; (iii) simplificar los procedimientos de despido colectivo; (iv) facilitar el uso de contratos temporarios (que incluyen las pasantías no remuneradas) y los acuerdos de trabajo por tiempo parcial; (v) limitar la extensión de la cobertura de convenios colectivos a nadie más que los signatarios directos; y (vi) ofrecer un uso más amplio a la aplicación de las cláusulas de los convenios colectivos. ${ }^{47}$

Sin embargo, como ha señalado reiteradamente el feminismo, las regulaciones del mercado laboral y los convenios colectivos proporcionan una protección crucial a los derechos de las mujeres en el entorno laboral. ${ }^{48}$

47 FMI, Informe de país de Argentina Núm. 17/409. 2017, 25.

48 Bürgisser, op. cit. (nota 8), 8. 
Lo que el análisis de impacto del FMI no hizo fue examinar de cerca el impacto combinado del paquete de reformas recomendado para Argentina en su totalidad. Es importante oponerse al tipo de modelo de evaluación de impacto llevado a cabo por el FMI y proponer alternativas. ${ }^{49}$ Los modelos basados en supuestos neoliberales tienden a apoyar recomendaciones de políticas neoliberales.

\section{Modos alternativos para evaluar el impacto de género de las políticas del FMI}

En el Reino Unido se ha recurrido a otra forma de estudiar el impacto de género del paquete de cambios en las políticas económicas en su totalidad frente a las políticas de austeridad que comenzaron en 2010. El análisis de impacto fue publicado por la Comisión de Igualdad y Derechos Humanos del Reino Unido (EHRC, por su sigla en inglés) ${ }^{50}$ y por el Grupo de Presupuestos de Mujeres del Reino Unido, ${ }^{51}$ que recurrieron a un modelo de microsimulación que mide el impacto inmediato de cambios en los impuestos, las transferencias monetarias (jubilaciones y pensiones, entre ellas) y el gasto en servicios públicos sobre los ingresos y beneficios en especie recibidos por diferentes individuos y hogares. Los modelos de este tipo están muy difundidos para representar los impactos distributivos de las políticas. No se construyen a partir del tipo de supuesto neoliberal de los modelos de equilibrio general dinámico y estocástico a los que recurre

\footnotetext{
49 Para otros análisis, ver Allison Corkery y Gilad Isaacs, "Human Rights Impact Assessments and the Politics of Evidence in Economic Policymaking", International Journal of Human Rights, 2020. Disponible en inglés en: https://doi.org/10.1080/13642987.2020.1804372

50 Jonathan Portes y Howard Reed, The Cumulative Impact of Tax and Welfare Reforms, (Comisión de Igualdad y Derechos Humanos, 2018); Howard Reed y Jonathan Portes, The Cumulative Impact on Living Standards of Public Spending Changes, (Comisión de Igualdad y Derechos Humanos, 2018).

51 Grupo de Presupuestos de Mujeres, Intersecting Inequalities. The Impact of Austerity on Black and Minority Ethnic Women in the UK, (Women's Budget Group, 2017). Este Grupo es una ONG feminista con sede en Londres.
} 
el FMI, basados en la operatoria de los mercados y la respuesta de las personas a los cambios en los precios y en el ingreso.

En el caso de las modificaciones a los impuestos directos (como el del ingreso y las contribuciones a la seguridad social) y las transferencias monetarias, el Reino Unido dispone de información oficial que permite un análisis sobre individuos y también hogares, desagregado en función de varias características. El análisis para la EHRC comprobó que, en promedio, las mujeres pierden más que los varones frente a los cambios en los impuestos directos y en las contribuciones. Las mujeres pierden un promedio de 400 libras esterlinas anuales y los varones no más de 30 libras esterlinas, si bien hay variaciones en función del estatus socioeconómico. Tanto mujeres como varones tienen ganancias en los grupos más adinerados (que se ubican en el $40 \%$ superior en la distribución del ingreso por hogares) pero las ganancias de las mujeres son menores que las de los varones. En el $60 \%$ de los hogares de menores ingresos, tanto mujeres como varones pierden, pero las mujeres pierden más que los varones, y sus pérdidas absolutas en efectivo son las más grandes del decil 2, el anteúltimo grupo en la escala de distribución del ingreso, en que las mujeres pierden 1500 libras esterlinas por año. También se analizaron variaciones por rango de edad, discapacidad o etnia. ${ }^{52}$ Se realizó un análisis equivalente por parte del Grupo de Presupuestos de Mujeres del Reino Unido, con especial hincapié en las mujeres de raza negra y de la minoría (Black and Minority Ethnic), ${ }^{53}$ y allí se encontró que las pérdidas más grandes las padecían las mujeres asiáticas pobres, seguidas de las mujeres negras pobres. ${ }^{54} \mathrm{El}$ FMI debe ser instado a producir un análisis similar para todos los programas de reformas de impuestos y transferencias que considera condiciones para sus préstamos.

\footnotetext{
52 Jonathan Portes y Howard Reed, op. cit. (nota 50).

53 "Black and Ethnic Minority" es una categoría official usada en el Reino Unido para desagregar los datos.

54 Grupo de Presupuestos de Mujeres, op. cit. (nota 51), 3.
} 
Otra dimensión igualmente importante de los programas de austeridad son los recortes al gasto en servicios públicos, que hace que menos personas tengan acceso a ellos. En el Reino Unido, los datos no permiten evaluar el impacto de estos recortes a nivel individual sino solamente a nivel de los hogares. Sin embargo, es posible distinguir los hogares entre sí según aquellas características relevantes a la igualdad de género, especialmente los factores que determinan las necesidades de cuidado de los hogares, por ejemplo, si se trata de hogares monoparentales o no, o con cuántos niños y niñas y la edad y el género de los adultos. El Grupo encontró que los hogares monoparentales compuestos por mujeres solas, que constituyen el $92 \%$ del total de las familias monoparentales, perderían un promedio de 4900 libras esterlinas en servicios. Las siguientes más afectadas eran las madres solteras que recibían una pensión. ${ }^{55} \mathrm{El}$ análisis de los recortes a los gastos realizado por la EHRC dividía los hogares en diecisiete categorías basada en la presencia o ausencia de niños y niñas, la cantidad de adultos y el género. Las pérdidas mayores se registraron en los hogares de tres niños/as o más y las menores, en los hogares sin niños/as. ${ }^{56}$ El FMI debe ser instado a producir un análisis similar de todos los recortes al gasto que considera como condiciones para sus préstamos. También se podrían analizar los recortes según su impacto sobre el empleo.

La economía feminista también ha producido maneras alternativas para evaluar el impacto del gasto sobre el cuidado infantil, y en ellas se utilizan modelos que no se basan en presupuestos neoliberales, no restringen el acceso al cuidado infantil al sector de trabajadores/as formales, y proponen la provisión de servicios de cuidado por parte del sector público, con fuerza de trabajo capacitada y bien remunerada. ${ }^{57}$ Este

\footnotetext{
55 Grupo de Presupuestos de Mujeres, op. cit. (nota 51), 29.

56 Howard Reed y Jonathan Portes, op. cit. (nota 50).

57 Jerome De Henau, Debbie Budlender, Fernando Filgueira, Ipek Ilkkaracan, Kijong Kim y Rafael Mantero, Investing in Free Universal Childcare in South Africa, Turkey and Uruguay:
} 
enfoque comienza por el costeo de la oferta de un servicio público de cuidado infantil basado en centros especializados, que recurre a una variedad de parámetros como: cuántos niños/as se inscriben y por cuánto tiempo, la proporción de niños/as frente a cuidadores/as y la remuneración y las condiciones laborales del lugar. Comienza por la situación del momento actual y permite contemplar la ampliación de las inscripciones, la mejora en la proporción niños/as-cuidadores/as y las mejoras de la remuneración y las condiciones laborales. La inversión total requerida para el sistema deseado puede computarse luego como una porción del PBI. Si se asume una inscripción universal y parámetros de lo más generosos, pero también factibles (de remuneración, proporción entre niños/as y personal, y capacitación y experiencia), la inversión anual requerida como porcentaje del PBI sería de 2,8 \% en Uruguay; 3,7\% en Turquía y 3,2 \% en Sudáfrica. Si se cobraran aranceles a las familias de ingresos más altos, la inversión neta requerida se vería reducida.

Es también posible evaluar cuántos puestos de trabajo se crearían con estas inversiones y la recaudación fiscal que se obtendría por los impuestos que pagarían los y las trabajadores/as que tomaran estos empleos. El empleo se genera por tres vías: creación directa de puestos de trabajo en el sector de cuidado infantil, creación indirecta de puestos de trabajo en la industria que provisiona al sector de cuidado infantil y puestos de trabajo inducidos que se desprenden del aumento del consumo producido por los ingresos del personal de cuidado infantil y de la provisión de la industria. Es posible cuantificar estas variables mediante un modelo de insumo-producto. La evaluación asume de forma realista que no hay empleo pleno, por lo tanto, los puestos laborales serán ocupados por mujeres y varones desempleadas/os o que no han podido sumarse al mercado laboral por

A Comparative Analysis of Costs, Short-term Employment Effects and Fiscal Revenue, (UN Women, 2019). Para un resumen de los resultados, ver la tabla 7.1. Disponible en inglés en https://www.unwomen.org/en/digital-library/publications/2019/07/discussion-paperinvesting-in-free-universal-childcare-in-south-africa-turkey-and-uruguay 
la falta de acceso a los servicios de cuidado infantil. La cantidad de puestos de trabajo generados para mujeres y varones puede estimarse con base en las proporciones actuales de mujeres y varones empleados en cada sector relevante. Aunque la mayoría de los puestos probablemente serán ocupados por mujeres, también habrá un incremento en los puestos laborales para varones, de manera que aumentará la tasa de empleo para ambos géneros, pero el aumento será mayor para mujeres que para varones, lo cual reducirá la brecha de empleo entre géneros.

\section{9. ¿Qué políticas debe apoyar el FMI para promover} la igualdad de género y concretar los derechos de las mujeres en Argentina? ¿Cómo la organización de las mujeres permitiría el reclamo en esa dirección?

El gobierno argentino se encuentra en estos momentos en un proceso de negociación con el FMI para renegociar los términos del acuerdo de endeudamiento provisto al gobierno anterior que marcó un hito en la propia historia del organismo: fue el préstamo de acceso especial más grande entregado jamás a un país (superando los 57000 millones de dólares). Esto sucede en un momento también peculiar de la historia, donde la economía sufre los impactos severísimos de la crisis global de la COVID-19. Se estima que, como consecuencia de este contexto, el PBI del país se habrá reducido más del $10 \%$ durante 2020.

En este contexto, todos los indicadores sociales han empeorado. Según datos del INDEC, la tasa de desocupación subió del 9,7\% en el tercer trimestre de 2019 a 11,7 \% en igual período de 2020 (último disponible). La incidencia de la pobreza por ingresos se elevó del 35,4\% de la población en el primer semestre de 2019 a 40,9 \% en el mismo período de 2020. En ese mismo lapso, la pobreza extrema subió de $7,7 \%$ a $10,5 \%$ de la población. Es de esperar que las brechas de género también se hayan profundizado. Dos indicadores que permiten confirmar esta intuición: i) mientras que la tasa de desocupación de los varones se elevó (entre el tercer trimestre de 2019 y el mismo 
período de 2020 ) de $8,9 \%$ a $10,6 \%$, la de las mujeres lo hizo de $10,8 \%$ a $13,1 \%$; ii) el $65,5 \%$ de los hogares del país declararon haber incrementado, durante la pandemia, el tiempo dedicado a las tareas domésticas y de cuidado no remunerado; en 64,1\% de estos hogares, esta tareas fueron asumidas por las mujeres de manera exclusiva o con una notoria mayor dedicación. ${ }^{58}$

La evidencia parece contundente para señalar que lo que menos necesita el gobierno argentino es un acuerdo que contenga las recetas habituales de austeridad, disciplina fiscal, y desregulación para mejorar la competitividad. ${ }^{59}$ Por el contrario, lo que se requiere es un Estado activo, que pueda disponer de recursos para fomentar la recuperación de actividades económicas que generen empleo de calidad y mejoren la remuneración al trabajo, a la vez que pueda preservar y expandir los programas de sostenimiento del ingreso de la población (incluyendo los variados programas de transferencias monetarias).

Como varias propuestas desde la mirada feminista han señalado, este puede ser también un tiempo donde la recuperación se oriente no solo a recuperar la situación pre-pandemia, sino a sentar las bases para una transformación estructural. ${ }^{60}$ En este marco, el FMI debería apoyar mecanismos que permitan al

58 INDEC, Estudio sobre el impacto de la COVID-19 en los hogares del Gran Buenos Aires. Agosto-octubre de 2020: Primer informe de resultados, (Instituto Nacional de Estadística y Censos, 2020).

59 Este es el tipo de condiciones que el FMI ha vuelto a imponer, por ejemplo, a Ecuador en el reciente acuerdo por 6500 millones de dólares, alcanzado en septiembre de 2020 en el marco del Servicio Ampliado. Allí se consigna que el gobierno ecuatoriano se compromete a "replegar las medidas relacionadas con la crisis conforme la economía empiece a recuperarse. La sostenibilidad fiscal estaría anclada al tope de la deuda del $57 \%$ del PIB para finales de 2025, según el Código Orgánico de Planificación y Finanzas Públicas (COPLAFIP) del país. La sostenibilidad fiscal estaría respaldada por una combinación de una reforma tributaria progresiva a mediano plazo y medidas de gasto que apuntan a alinear a Ecuador con a sus pares regionales". (https://www.imf.org/es/News/Articles/2020/10/01/pr20302-ecuadorimf-executive-board-approves-27-month-extended-fund-facility).

60 Ver, por ejemplo, Action Aid, Another World is Possible: Advancing Feminist Economic Alternatives to Secure Rrights, Justice and Autonomy for Women and a Fair, Green, Genderequal World, (Action Aid, 2020); Cepal-ONU Mujeres, Cuidados en América Latina y el Caribe 
gobierno argentino atender y desarmar nudos básicos de reproducción de la desigualdad. Tres ejes de acciones podrían respaldarse en este sentido. En primer lugar, la inversión en una variedad de políticas que sienten las bases de un sistema integral de cuidados (de hecho, el gobierno argentino ya se encuentra avanzando con una agenda en este sentido). ${ }^{61}$ Estas políticas deberían expandirse más allá de la clásica promoción de licencias vinculadas con el cuidado y la expansión de espacios para el cuidado infantil, para incluir el mejoramiento de la infraestructura física para el cuidado, incluyendo el transporte y la seguridad urbana, así como también mecanismos de profesionalización y mejoramiento en las condiciones laborales de las personas trabajadoras del cuidado.

En segundo lugar, el sostenimiento de programas de transferencias monetarias (hoy altamente feminizados), impulsando su transformación para remover sus sesgos de género (como los operados a través de las condicionalidades vinculadas al cuidado) y potenciar sus impactos positivos en las condiciones de vida. ${ }^{62}$

En tercer lugar, el impulso de políticas sectoriales que promuevan un cambio en la matriz productiva, facilitando una transición desde una estrategia de desarrollo basada en el extractivismo, hacia una estructurada en torno a la provisión de servicios esenciales para la reproducción cotidiana de la vida, que promueva formas más sustentables de proveer a las necesidades humanas, y favorezca la innovación y la creatividad. Dado que esta transición, en un país como Argentina, que es dependiente para su ahorro externo de las exportaciones de origen minero y agroindustrial, puede limitar el acceso a divisas, lo que debería ser tenido en cuenta a la hora de pactar las nuevas condiciones

en tiempos de Covid19: Hacia sistemas integrales para fortalecer la respuesta y la recuperación (CEPAL-ONU Mujeres, 2020).

61 Ver al respecto https://www.argentina.gob.ar/generos/cuidados.

62 Al respecto, en Argentina parece haber una retracción con la eliminación del Ingreso Familiar de Emergencia (IFE) en el contexto de la segunda ola de la COVID-19 y las medidas restrictivas que requiere. 
del acuerdo. Más aún, el FMI podría considerar la reducción de deuda a cambio de la expansión de actividades productivas que mitiguen el proceso de cambio climático.

El movimiento de mujeres y feminista en Argentina, que se ha transformado en los últimos años en el movimiento social más vibrante y transformador, podría ser un actor relevante en el reclamo por esta actitud renovada por parte del FMI. De hecho, el tema del endeudamiento ya ha calado en la agenda feminista. Vale como ejemplo la expansión de la consigna originaria del movimiento Ni Una Menos (ni una menos, vivas nos queremos), que en los últimos $8 \mathrm{M}$ y en el marco de los paros internacionales de mujeres se convirtió en "ni una menos, vivas, libres y desendeudadas nos queremos".

Es que la experiencia del endeudamiento (público y privado) no es ajena a la vida de las mujeres. Por el contrario, el extractivismo financiero se percibe en los cuerpos de las mujeres. Problematizar la dinámica abstracta de las finanzas implica reconocerla en su relación con la vida cotidiana, con las formas de la violencia en los hogares y en los diversos territorios y con las modalidades actuales de explotación del trabajo. ${ }^{63}$ Las implicancias del endeudamiento sobre el goce efectivo de los derechos económicos, sociales y culturales se vienen trabajando desde el feminismo en relación con el endeudamiento de las economías domésticas, pero es extrapolable al endeudamiento de los países (que no es más que el endeudamiento de la población que los habita).

La necesidad de destinar recursos al pago de la deuda implica restarlos para la provisión de servicios esenciales para la vida. Que el acreedor tenga además la potestad de exigir condicionalidades, implica limitar el espacio y la autonomía de las políticas públicas. De hecho, esta traslación en el abordaje feminista de la deuda desde lo doméstico a la deuda soberana, se puede expresar en el involucramiento de organizaciones de mujeres en el juicio

63 Lucía Cavallero y Verónica Gago, Una lectura feminista de la deuda, (Fundación Rosa Luxemburgo, 2019). 
popular a la deuda y al FMI llevado a cabo durante el año 2020, en el que se fueron recopilando evidencias del impacto concreto de la deuda hasta llegar a un veredicto que convoca a la suspensión del pago y la investigación del proceso de endeudamiento. ${ }^{64}$

Más allá del valor simbólico de este juicio popular, este movimiento pretende generar conciencia y demanda social para crear mecanismos de control ciudadano de los procesos de endeudamiento, que involucren, entre muchas otras cosas, la obligación de estudios de evaluación de impacto social, ambiental, de género y sobre los derechos humanos, como condición para aceptar nuevos empréstitos y todas sus consecuencias.

\section{Conclusiones}

El FMI ya no es ajeno a las cuestiones de género. Al igual que otras organizaciones financieras internacionales, que muchos gobiernos y que instituciones regionales y multilaterales, ha pasado de la invisibilización de la cuestión, a aceptarla como un asunto macroeconómico crítico.

Así, el FMI ha ido construyendo una narrativa de género que, sin embargo, como hemos intentado mostrar a lo largo de este artículo, resulta estrecha e instrumental, y no contribuye en la práctica a promover los derechos humanos de las mujeres. ${ }^{65}$

La visión de género del FMI se sustenta en la idea que reducir las brechas de género y promover la participación económica de las mujeres resulta beneficioso en términos económicos,

64 Sobre el proceso de juicio popular y el veredicto, ver https://autoconvocatoriadeuda. blogspot.com/2020/12/fallo-del-tribunal-del-juicio-popular.html

65 Cabe notar, asimismo, que la visión de género del FMl es binaria, en términos de enfatizar la situación de las mujeres a la vez que invisibiliza la de la población LGTBIQ+. Si bien no hemos profundizado en este aspecto a lo largo del texto, las inconsistencias que señalamos respecto a promover los derechos de las mujeres a la vez que se sostienen las recetas convencionales de disciplina fiscal, podrían extenderse para pensar la situación de las personas de los colectivos de la diversidad, y el riesgo que tendría el sostenimiento de las medidas de acción afirmativa para esta población. Por ejemplo, las dificultades de implementar el cupo laboral trans en la administración pública en un contexto de recortes fiscales. 
porque puede derivar en mayor eficiencia y productividad y, en consecuencia, en mayor crecimiento económico.

Pero aun en esta visión estrecha, también se verifican contradicciones, que fueron detalladas a lo largo de cada una de las secciones. Si bien el FMI considera importante llevar adelante acciones que promuevan la participación económica de las mujeres, no se preocupa por los derechos laborales de las mismas. Más aún, en el mismo acuerdo de entendimiento entre el FMI y un determinado país, pueden encontrarse recomendaciones para facilitar la participación laboral de las mujeres, junto con condicionalidades vinculadas a reformas laborales flexibilizadoras. Como lo demuestra el caso argentino analizado en particular, un mismo acuerdo con el FMI puede incluir un capítulo de género que promueva políticas públicas para reducir las brechas de género, y un capítulo fiscal que le prive al país de los recursos para poder llevarlas a cabo.

De la misma manera, el reconocimiento instrumental del trabajo doméstico y de cuidados no remunerado, entendido sólo como un obstáculo para el crecimiento económico, no le permite al FMI reconocer el rol económico sistémico de este trabajo, y la forma injusta de la organización social del cuidado. Así, se limita a sugerir intervenciones acotadas (como por ejemplo la expansión de los servicios de cuidado infantil), que a su vez pretende hacer compatible con la austeridad fiscal.

La mirada instrumental también se traduce en las iniciativas de presupuestos sensibles al género promovidas desde el FMI. Las mismas promueven análisis focalizados en intervenciones específicas del presupuesto, en lugar de promover el estudio del impacto de género del presupuesto en su totalidad. De esta forma, se promueve identificar partidas que pueden tener efectos positivos sobre la vida de las mujeres y las brechas de desigualdad, pero se omite contrastarlas con el impacto que pueden tener las lógicas macrofiscales que se imponen.

Esta visión "contradictoria" se verifica cuando se mira el discurso y las acciones que el FMI (al igual que otras organizaciones 
financieras internacionales) han tomado en el contexto de la crisis del COVID-19. Por un lado puede señalarse que la narrativa de género del FMI no se vio modificada, y mantiene su perfil instrumental, aunque lo decora indulgentemente en el actual contexto. Por ejemplo, Gita Gopinath, Economista Jefa del FMI, declaró en su discurso del 8 de marzo de 2021 en la inauguración de la cátedra Dr. Hansa Mehta:

El argumento moral por la igualdad de género ya está claro. Hoy, voy a enfatizar que nosotros en el FMI nos hemos esforzado por hacer que el argumento económico a favor de la igualdad de género también quede muy claro. [...] Al tiempo que los países alrededor del mundo luchan por hacer crecer sus economías [...] aprovechar el enorme potencial de las mujeres es, sin lugar a dudas, algo que beneficia tanto al empoderamiento de las mujeres como al crecimiento económico mundial inclusivo. ${ }^{66}$

Adicionalmente, la distancia entre la narrativa de género y el impacto concreto en la vida de las mujeres se vuelve a manifestar en el contexto de la actual crisis. Para el caso de América Latina, tanto el incremento del endeudamiento de los países, como la persistencia de un abordaje económico ortodoxo por parte del FMI, puesto de manifiesto en las condicionalidades de sus créditos y en las recomendaciones de política macroeconómica, dan cuenta que ningún cambio significativo ha ocurrido en esta institución y en el potencial impacto de sus acciones. ${ }^{67}$

Lo que está detrás de la narrativa de género del FMI es la resistencia a vincular los niveles micro, meso y macro de análisis. Como se señaló a lo largo de este artículo, desde el FMI se propone que los problemas de la participación laboral de las mujeres son de ellas (y que hay que ayudarlas a superarlos), y

\footnotetext{
66 Tomado de: https://www.imf.org/en/News/Articles/2021/03/08/sp030821-gita-gopinathinaugural-dr-hansa-mehta-lecture. Traducción propia.

67 Juan Pablo Bohoslavsky y Mariana Rulli, “Bretton Woods' Pandemic Policies: A Gender Equality Analysis_-Perspectives from Latin America", 1 Development, 2021, Vol. 64.
} 
no un problema sistémico que requiere por un lado una revisión de las estrategias de desarrollo basadas en modelos que no generan empleo de calidad (sino que más bien empujan a la baja todos los estándares laborales) y, por el otro, políticas públicas activas que contribuyan a remover las barreras que enfrentan las mujeres y que no pueden implementarse en el marco de exigencias de austeridad y otras políticas económicas ortodoxas.

Finalmente, los intentos cuantitativos del FMI de realizar análisis más integrados, que evalúen el impacto de género de las políticas económicas, fracasan desde sus propios diseños, al apoyarse en supuestos neoliberales sobre los procesos de toma de decisiones de las personas y el funcionamiento de los mercados. Como la Economía Feminista lo ha dicho ya extensamente, este tipo de modelización sirve cada vez menos para explicar la realidad (porque entre otras cosas omite considerar las relaciones sociales de poder que determinan el accionar de las personas), y más aún para poder predecir lo que pasará.

También como lo demuestra la Economía Feminista y como se menciona a lo largo de este artículo, existen alternativas. Como es el caso de los análisis de impacto basados en información micro que permite reconocer desde la evidencia concreta (y no desde supuestos teóricos neoliberales) la variedad de respuestas posibles de las personas a partir de sus distintas características y de las de los hogares en las cuales viven.

Asimismo, existe la posibilidad de gestar, desde la organización y movilización feminista, conciencia social sobre las implicancias que los procesos de endeudamiento tienen sobre la vida concreta de las personas. Y con ello, generar una demanda social que dispute las narrativas y que exija monitoreo ciudadano sobre los procesos de toma de decisión en torno a las deudas soberanas. 